\title{
Three-dimensional self-assembly of metal nanoparticles: Possible photonic crystal with a complete gap below the plasma frequency
}

\author{
Zhenlin Wang, ${ }^{1,2}$ C. T. Chan, ${ }^{1}$ Weiyi Zhang, ${ }^{2}$ Naiben Ming, ${ }^{2}$ and Ping Sheng ${ }^{1}$ \\ ${ }^{1}$ Department of Physics, Hong Kong University of Science and Technology, Clear Water Bay, Kowloon, Hong Kong, China \\ ${ }^{2}$ National Laboratory of Solid State Microstructures, Nanjing University, Nanjing 210093, China
}

(Received 25 May 2001; published 31 August 2001)

\begin{abstract}
We study theoretically the optical properties of a three-dimensional self-assembly of spherical-metal nanoparticles. Our band-structure calculations show that photonic band gaps in near infrared and optical frequencies can be realized in a fcc lattice of metal particles with radii of approximately $160 \mathrm{~nm}$. When absorption is taken into account, we found that the gap is preserved in good metals like silver. The metal nanoparticles can be replaced by dielectric spheres coated with a layer of good metal.
\end{abstract}

DOI: 10.1103/PhysRevB.64.113108

PACS number(s): 42.70.Qs, 78.67.Bf

Photonic crystals are three-dimensional periodic dielectric structures, with lattice constants of the order of the desired electromagnetic (EM) wavelength of operation. The free-EM wave dispersion in such crystals is greatly modified due to the presence of periodicity and refractive-index contrast. Under favorable circumstance, a photonic band gap (PBG) in the dispersion relation can open up, in which light cannot propagate in any direction. ${ }^{1}$ Such a structure is believed to have a deep impact on a wide range of photonic applications. $^{2-5}$

Recently, considerable progress has been made in constructing three-dimensional photonic crystals with submicrometer periodicity, using the sophisticated "top-down" fabrication techniques, ${ }^{6}$ the self-assembly of monodispersed microspheres and related infiltration method, ${ }^{7}$ and the holographic lithography. ${ }^{8}$ Compared to the micromachining methods for the production of PBG crystal for visible wavelengths, the self-organization process is a very cheap and relatively easy method too.

On the other hand, the interaction of light with nanoscale metal particles has been the subject of extensive research. ${ }^{9}$ Researchers have used metal nanoparticles as building blocks for novel materials. ${ }^{10-14}$ We will show in this paper that metallic nanoparticles can be used as building blocks for photonic crystals operating at near IR and optical frequencies. It is not intuitively obvious that metallic nanoparticles can serve the purpose. First, metals are dispersive and can be rather absorbing at IR and optical frequencies. Second, it is very difficult to fabricate metal nanoparticles that are monodispersed spheres with radii of the order of $150 \mathrm{~nm}$, the scale length needed to realize photonic gaps at optical frequencies. We are going to show that both of these problems can be solved. First, our calculations will show that the absorption problem can be alleviated by a careful choice of the metal component. In order to build a convincing case, we will use experimentally measured dielectric constants for the frequency range of interest (in contract to Drude or Drude-like models that are used frequently in the literature $)^{15}$ in all of our calculations. We will also suggest a simple and practical way (using metal coated silica spheres) to bypass the need of using metal nanoparticles of large $(150 \mathrm{~nm})$ radius.

We will draw our conclusions from results that are based on band-structure calculations employing periodic boundary conditions and multiple-scattering technique, ${ }^{16}$ as well as an "on-shell" method ${ }^{17}$ that gives directly transmission/ reflection/absorption coefficients for a slab of finite thickness. The band-structure code sets the imaginary part of the dielectric constants to zero, which allows us to determine whether a photonic band gap exists or not in the absence of absorption. The slab calculations consider explicitly both the real and imaginary parts of the dielectric constants, modeling the metal faithfully according to published complex dielectric constants, and will help us to see what absorption will do to the band gap. We will consider three common metals in our calculations: silver, copper, and nickel. Our bandstructure calculations show that fairly large photonic band gaps are obtained in optical frequencies for all the metals we have considered. The band gaps are the consequence of a large and negative real part of the dielectric constant, a condition that is easily satisfied by most metals up to IR and even optical frequencies. However, when absorption is taken into account, we found that the gap survives in good metals like silver, but are seriously compromised in nickel. We emphasize again that the complex dielectric constants are taken from experimental measurements ${ }^{18}$ for all the metals in all of our calculations. We shall focus on the face-centeredcubic(fcc) arrangement of spheres, which is the structure usually obtained in self-assembly processes of spheres. We will see later (in Fig. 2) that the size of the photonic gaps increases with the filling ratio of metal nanospheres, but we are going to assume a $66 \%$ filling ratio for almost all of the calculations although the filling ratio can reach $74 \%$ (closepacking case) for a fcc structure. This is because in experimental implementations, we expect that the metal spheres would be coated with protective inert coatings to avoid oxidation. A lower-filling ratio of the metal spheres also cuts down absorption.

From tabulated values in Ref. 19, the plasma frequencies for silver, copper, and nickel are found to be 9.0, 7.4, and 4.9 $\mathrm{eV}$, respectively, so that the real part of the dielectric constant of metals has the property of being negative and large in magnitude for wavelengths in near IR and optical frequencies that lie well below the plasma energies. This large dielectric contrast should be favorable for the opening of a PBG in these frequencies. This is illustrated in Fig. 1, where we display the band structures of fcc arrays of spherical- 

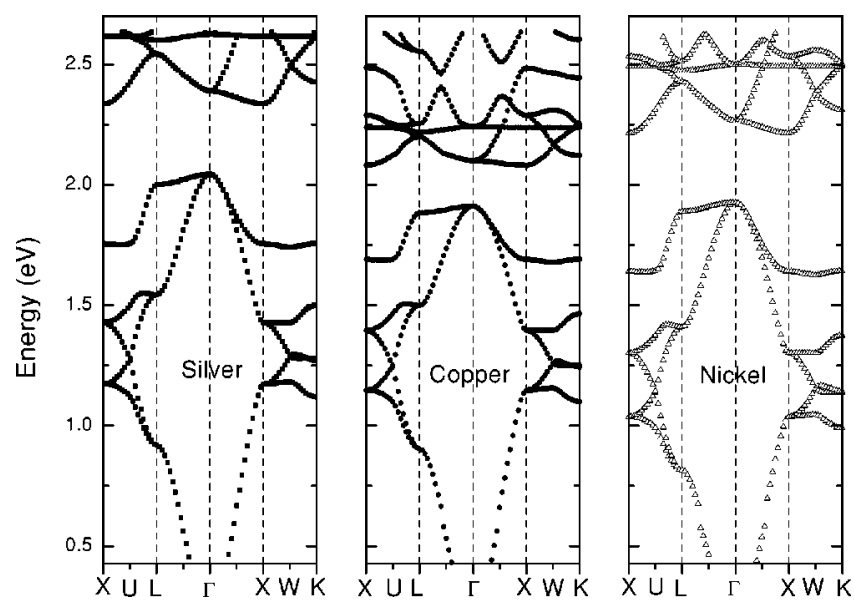

FIG. 1. Calculated band structure for fcc photonic crystals consisting of spherical-metal nanoparticles with radius $r=160 \mathrm{~nm}$ for (a) silver, (b) copper, and (c) nickel. All structures have the same filling ratio $f=0.66$. In the band-structure calculations, the imaginary part of the dielectric constant is set to zero.

metal nanoparticles in air with particle radius $r=160 \mathrm{~nm}$. The filling ratio of the metal component is $f=0.66$ for all metals. We found that all the metallo-dielectric(MD) nanostructures possess a large PBG between the fifth and sixth bands. As the PBG opens as a result of the real part of dielectric constant $\epsilon_{r}$ being large and negative, we expect similar effect in self-organizations of other metal nanoparticles such as gold and aluminum. We found that the gap edges

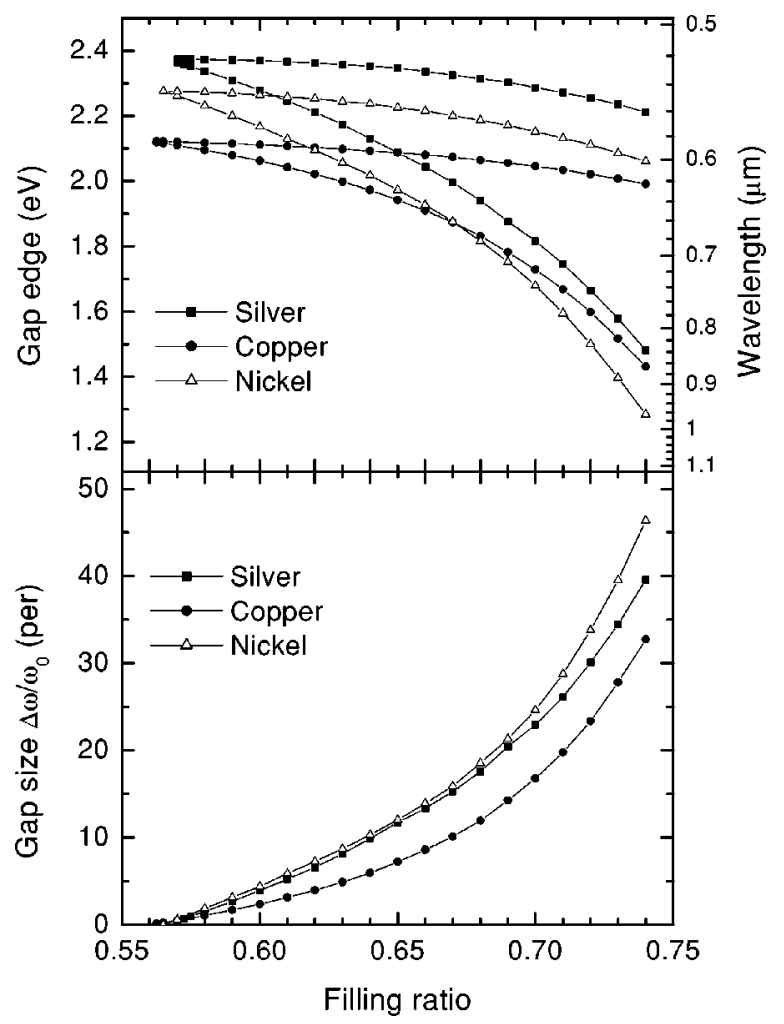

FIG. 2. Dependence of the gap position and gap-width/midgap frequency as functions of filling ratio for fcc photonic crystals for silver, copper, and nickel nanoparticles with radius $r=160 \mathrm{~nm}$.

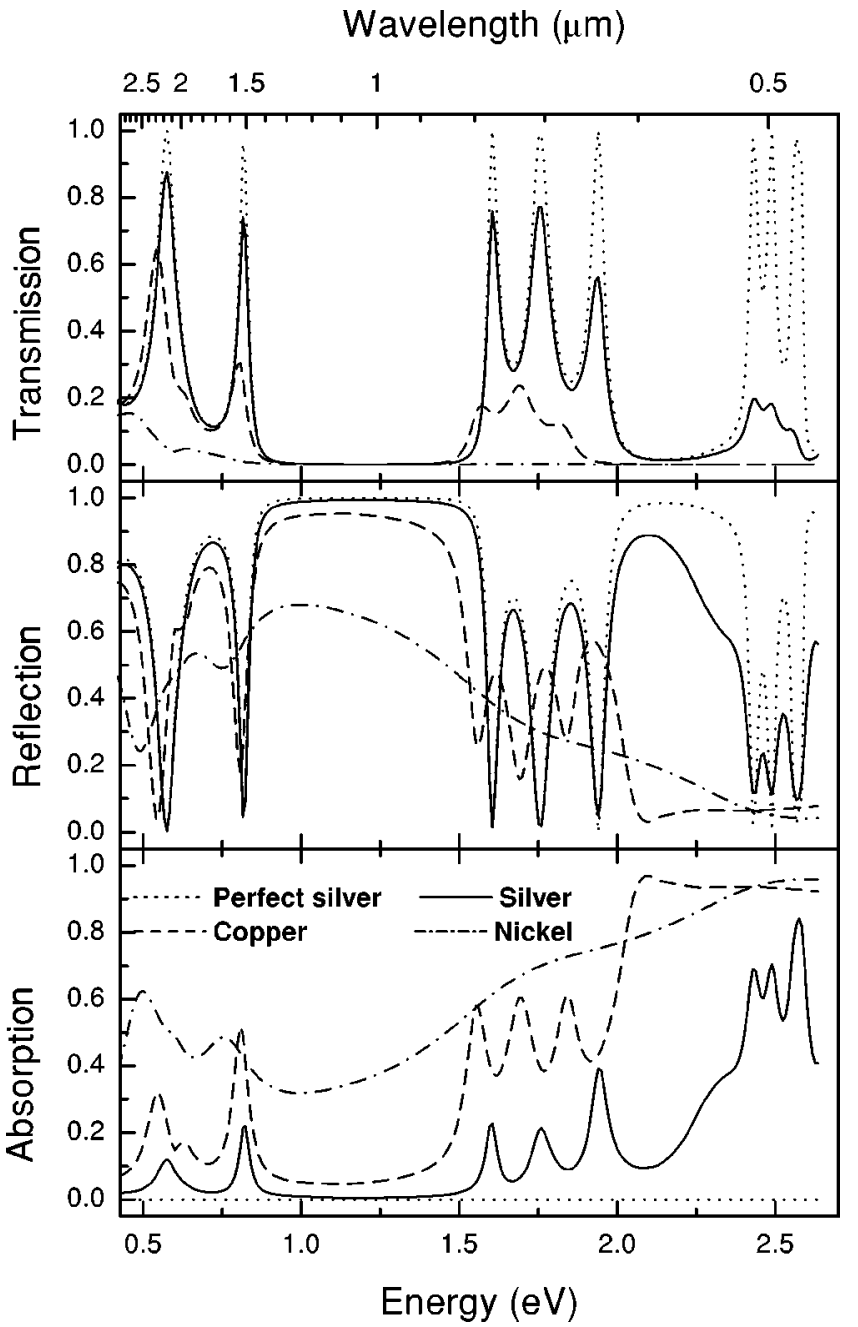

FIG. 3. Calculated transmission, reflection, and absorption by four layers of (111) orientated fcc photonic-crystal slabs for silver, copper, and nickel nanoparticles with radius $r=160 \mathrm{~nm}$. Experimental complex dielectric constants are used. For comparison, the spectra of a photonic-crystal slab composed of "nonabsorbing" silver spheres $[\operatorname{Imag}(\epsilon)=0]$ are also shown

show some variations for silver, copper, and nickel as a consequence of their different magnitude and dispersion relations of the dielectric constant in these frequencies. A small region of distorted dispersion in the lower part of the figure is observed for copper and nickel due to the mismatch of dielectric constants in the optical and IR frequencies tabulated from separate experiments. ${ }^{18}$

The frequency and size of the photonic gap depend on both the filling ratio and the radius of the spheres. We found that silver spheres with $r=160 \mathrm{~nm}$ has the largest gap in optical frequencies for a filling ratio of $f=0.66$. We now consider the dependence of PBG position and gap-width/ midgap frequency as functions of filling ratio of the metal spheres with a fixed radius $r=160 \mathrm{~nm}$. Results are shown in Figs. 2(a) and 2(b). A PBG emerges when the volume fraction of the metal component exceeds a certain critical value, and increases as a function of the filling ratio. The critical volume fraction is seen to be nearly the same for all metals. The gap width reaches its maximum for the close-packing 


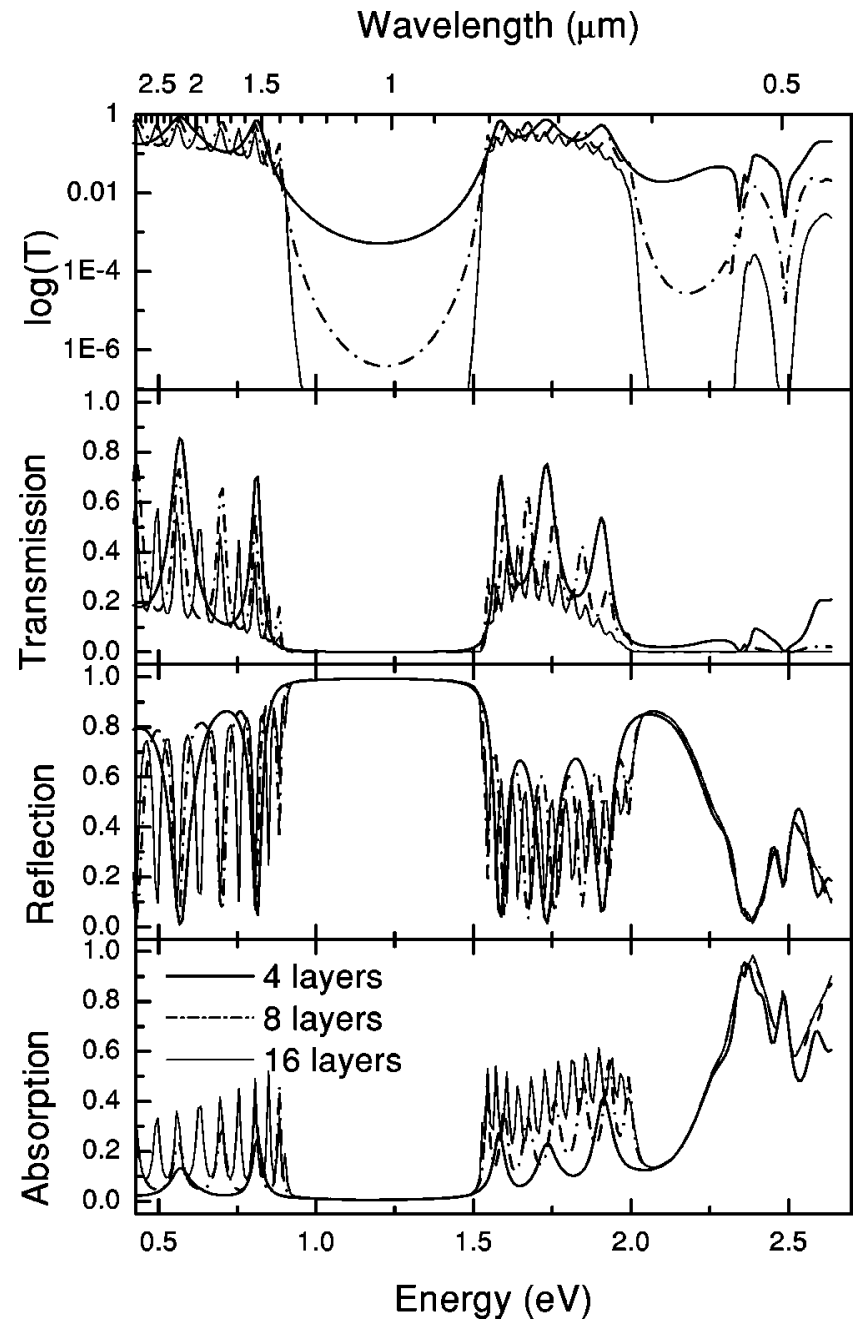

FIG. 4. Calculated transmission, reflection, and absorption by four, eight, and 16 layers of a (111) orientated slab of a fcc lattice of silver-coated silica nanoparticle. The silver shell has a thickness of $40 \mathrm{~nm}$ and the silica core has a radius of $120 \mathrm{~nm}$.

case $(f=0.74)$. Although we get bigger gaps for higherfilling fractions, it is most likely that a lower-filling ratio (e.g., 66\%) will be used experimentally for aforementioned reasons.

In band-structure calculations, only the real part of the dielectric constant is used since the size of the photonic band gap can be precisely defined only in the absence of absorption. It is important to know how the features and the properties of the PBG would be affected by material absorption. In the microwave and far-infrared region, metal behaves almost like a perfect conductor, and photonic crystals incorporated with metal scatterers have been studied at these low frequencies. ${ }^{20}$ From near IR down to ultraviolet, absorption must be considered carefully. In the slab calculations we are going to describe, which give transmission/reflection/ absorption spectra, the material absorption is included naturally by turning on the imaginary part of the complex (experimentally measured) dielectric constant $\epsilon$.

We have calculated the transmission, reflection, and absorption spectra through a slab of four layers of a (111)oriented MD photonic crystal, which displays a PBG in the visible region from the band-structure calculation as shown in Fig. 1. Results for silver, copper, and nickel are compared and the transmission, reflection, and absorption of light by the thin slabs are plotted in Figs. 3(a), 3(b), and 3(c), respectively. For comparison with the band structure, the spectra for the nonabsorbing silver (imaginary part of $\epsilon$ set to zero) particles are also shown. Band structure shows there is a directional gap from $0.92 \mathrm{eV}$ to $1.54 \mathrm{eV}$ and an absolute gap from $2.04 \mathrm{eV}$ to $2.40 \mathrm{eV}$ along the $\Gamma L$ direction. This is almost perfect correspondence between the band-structure result and the calculated transmission spectrum for "nonabsorbing" silver-photonic crystal, which shows two pronounced dips (almost 100\% reflectance) centered at $1.21 \mathrm{eV}$ and $2.19 \mathrm{eV}$, respectively. When the effect of absorption is included by turning on the imaginary part of $\epsilon$, noticeable changes in the transmission/reflection spectra are observed, especially for nickel in the high-energy region. Generally speaking, absorption is small for longer wavelengths and increases with frequency. For silver particles, the reflection within the directional gap remains almost the same as that of the "nonabsorbing" case; while it shows a notable decrease near the blue edge of the higher stop gap. Nevertheless, a maximum reflection of $90 \%$ can still be achieved in the stop gap. An increase in the slab thickness can lead to a small increase in the reflection within the stop gap, but the reflection coefficient saturates quickly for further increase in the number of layers.

For nickel, the pass bands at higher frequencies predicted by the band structure calculations for "nonabsorbing" nickel spheres become a region of strong absorption when the imaginary part of the dielectric constant is taken into account. The absorption is strong enough so that the edges of the photonic band gap are smeared out. The higher-frequency stop gap is totally destroyed, and even the lower-frequency directional gap is hardly discernible as the band edges are smoothed by the absorption. Since photonic band-gap originates from the collective scattering effect of many spheres, the gap cannot be well established if light cannot reach the deeper layers due to strong absorption. Thus it would be difficult to observe any photonic-gap effect in nanostructured crystals made of nickel nanoparticles. For MD crystals consisting of copper particles, the directional gap is still fairly well-defined both in the reflection and transmission spectra; while the reflection within the stop gap reduces to a small peak adjacent to the peaks resulting from the interlayer interference. Our calculations suggest that both the directional gap in the infrared and the PBG in the visible region are observable for MD photonic-crystal built with good-metal nanoparticles.

We note that a PBG around the plasma frequency has been found recently for a fcc lattice of spherical scatterers with a real Drude-like dielectric function by Moroz. ${ }^{21}$ As the dielectric constant changes sign across the plasma frequency in a real-Drude model, it can be arbitrarily small near the plasma frequency, producing a large dielectric contrast relative to the embedding dielectric medium, which results in the PBG. Compared to Moroz's PBG, our predicted PBG opens in the frequencies where the real part of the dielectric constant $\epsilon_{r}$ is large and negative. 
Current technology produces metal nanoparticles that are typically smaller than $150 \mathrm{~nm}$ in radius. ${ }^{9,14}$ These are too small for the construction of a MD PBG in the visible region. However, we can bypass this hurdle by using metal-coated spheres instead of solid-metal nanoparticles. As the penetration depth of a good metal is of the order of several tens of nanometer, coating a thin metal layer on monodispersed dielectric particles should work as solid-metal nanoparticles do. This is illustrated in Fig. 4, where we show the transmission, reflection, and absorption spectra of a fcc array of silver-coated dielectric nanoparticles. The coated particle consists of a silver shell with a thickness of $40 \mathrm{~nm}$ and a silica core with a radius of $120 \mathrm{~nm}$. The dielectric constant of silica is chosen to be $\epsilon=2.1$ as dispersion and absorption of silica at optical frequencies can be neglected. We found that the spectra are almost the same as solid-silver nanoparticle. Several methods have been developed to synthesize these composite nanoparticles that consist of a dielectric-core coated with a metallic shell. ${ }^{22-24}$ In particular, the thickness of the metal shell can be controlled fairly, precisely. ${ }^{22}$

In Fig. 4, we also compare the spectra for photonic crystal slabs with four, eight, and 16 layers of coated spheres. We see from this figure that the photonic gaps are already very well established for as little as four layers. The transmission in the pass bands diminishes as the number of layer increases, as it should be, but the transmission/reflection/ absorption within the photonic gap quickly saturates with the slab thickness. These metal nanoparticle derived systems are such potent photonic band-gap systems that a very thin slab (four to eight layers) should be good for most purposes.

In short, we showed that spherical-silver nanoparticles of radii of about $160 \mathrm{~nm}$ are plausible building blocks for selfassembled photonic crystals operating at optical frequencies. The difficulty of fabricating such particles can be bypassed by using silver-coated silica spheres, with the added advantage that the frequency of the PBG can be tuned simply by changing the size of the silica core.

This work is supported in part by RGC through HKUST6145/99P and the key research project in "Climbing Program" by the National Science and Technology Commission of China. Z. W. acknowledges partial financial support from the Natural Science Foundation of China (NSFC). W. Z. also acknowledges partial support from NSFC under "Excellent Youth Foundation."
${ }^{1}$ E. Yablonovitch, Phys. Rev. Lett. 58, 2059 (1987).

${ }^{2}$ J.D. Joannopoulos, P.R. Villeneuve, and S. Fan, Nature (London) 386, 143 (1997).

${ }^{3}$ S. John and J. Wang, Phys. Rev. B 43, 12772 (1991).

${ }^{4}$ S.-Y. Lin et al., Science 282, 274 (1998).

${ }^{5}$ T.F. Krauss and R.M. De La Rue, Prog. Quantum Electron. 23, 51 (1999).

${ }^{6}$ S. Noda and A. Sasaki, Jpn. J. Appl. Phys. 36, 1907 (1997); S.-Y. Lin et al., Nature (London) 394, 251 (1998).

${ }^{7}$ B.T. Holland, C.F. Blanford, and A. Stein, Science 281, 538 (1998); J.E.G. Wijnhoven and W.L. Vos, ibid. 281, 802 (1998); A.A. Zakhidov et al., ibid. 282, 897 (1998); S.H. Park and Y. Xia, Adv. Mater. 10, 1045 (1998); P.V. Braun and P. Wiltzius, Nature (London) 402, 603 (1999); J.F. Bertone et al., Phys. Rev. Lett. 83, 300 (1999); A. Blanco et al., Nature (London) 405, 437 (2000).

${ }^{8}$ M. Campbell et al., Nature (London) 404, 53 (2000).

${ }^{9}$ U. Kreibig and M. Vollmer, Optical Properties of Metal Clusters (Springer, Berlin, 1995).

${ }^{10}$ A. Taleb, V. Russier, A. Courty, and M.P. Pileni, Phys. Rev. B 59, 13350 (1999).

${ }^{11}$ Z.L. Wang et al., Adv. Mater. 10, 808 (1998).

${ }^{12}$ M. Brust et al., Langmuir 14, 5425 (1998).

${ }^{13}$ M. Aindow et al., Philos. Mag. Lett. 79, 569 (1999).

${ }^{14}$ A.K. Boal et al., Nature (London) 404, 746 (2000).

${ }^{15}$ A.R. McGurn and A.A. Maradudin, Phys. Rev. B 48, 17576
(1993); V. Yannopapas, A. Modinos, and N. Stefanou, ibid. 60, 5359 (1999); I. El-Kady et al., ibid. 62, 15299 (2000).

${ }^{16}$ J. Korringa, Physica (Utrecht) 13, 392 (1947); W. Kohn and N. Rostoker, Phys. Rev. 94, 1111 (1954).

${ }^{17}$ J.B. Pendry, J. Mod. Opt. 41, 209 (1993); N. Stefanou, V. Karathanos, and A. Modinos, J. Phys.: Condens. Matter 4, 7389 (1992); K. Ohtaka and Y. Tanabe, J. Phys. Soc. Jpn. 65, 2276 (1996).

${ }^{18}$ Optical freqencies data from ultraviolet to near IR $(0.64 \mathrm{eV})$ are taken from P.B. Johnson and R.W. Christy, Phys. Rev. B 6, 4370 (1972); for $\mathrm{Ag}$ and $\mathrm{Cu}$; 9, 5056 (1974); for Ni; IR data below 0.64 eV from Ref. 19.

${ }^{19}$ M.A. Ordal et al., Appl. Opt. 22, 1099 (1983).

${ }^{20}$ D.R. Smith et al., Appl. Phys. Lett. 65, 645 (1994); E.R. Brown and O.B. McMahon, ibid. 67, 2138 (1995); E. Ozbay et al., ibid. 69, 3797 (1996); K.A. McIntosh et al., ibid. 70, 2937 (1997); D.F. Sievenpiper, M.E. Sickmiller, and E. Yablonovitch, Phys. Rev. Lett. 76, 2480 (1996); S. Fan, P.R. Villeneuve, and J.D. Joannopoulos, Phys. Rev. B 54, 11245 (1996); J.B. Pendry, J. Phys.: Condens. Matter 8, 1085 (1996); W.Y. Zhang et al., Phys. Rev. Lett. 84, 2853 (2000).

${ }^{21}$ A. Moroz, Phys. Rev. Lett. 83, 5274 (1999); Europhys. Lett. 50, 466 (2000).

${ }^{22}$ S.J. Oldenberg et al., Chem. Phys. Lett. 288, 243 (1998).

${ }^{23}$ C.-W. Chen, T. Serizawa, and M. Akashi, Langmuir 15, 7998 (1999).

${ }^{24}$ W. Wen et al., Phys. Rev. Lett. 82, 4248 (1999). 